\title{
INTERIOR PENALTY CONTINUOUS AND DISCONTINUOUS FINITE ELEMENT APPROXIMATIONS OF HYPERBOLIC EQUATIONS.
}

\author{
ERIK BURMAN, ALFIO QUARTERONI, AND BENJAMIN STAMM
}

\begin{abstract}
In this paper we present the continuous and discontinuous Galerkin methods in a unified setting for the numerical approximation of the transport dominated advection-reaction equation. Both methods are stabilized by the interior penalty method, more precisely by the jump of the gradient in the continuous case whereas in the discontinuous case the stabilization of the jump of the solution and optionally of its gradient is required to achieve optimal convergence. We prove that the solution in the case of the continuous Galerkin approach can be considered as a limit of the discontinuous one when the stabilization parameter associated with the penalization of the solution jump tends to infinity. As a consequence, the limit of the numerical flux of the discontinuous method yields a numerical flux for the continuous method too. Numerical results will highlight the theoretical results that are proven in this paper.
\end{abstract}

\section{INTRODUCTION}

The discontinuous Galerkin finite element method (DGFEM) was introduced by Reed and Hill in 1973 for the neutron transport equation [28]. They compared the DGFEM with the continuous Galerkin finite element method (CGFEM) by means of numerical experiments. In their examples they highlighted the good stability properties of the DGFEM. The first analysis was performed a year later by Lesaint and Raviart [23]. A sharpened analysis using the stronger stability of the DGmethod was proposed in the 1980s by Johnson et al [20]. More recently, Houston, Schwab and Süli [17] presented an $h p$-analysis for the upwind DGFEM applied to advection-diffusion-reaction equations, while Brezzi, Marini and Süli [5] generalized the upwind DGFEM by replacing the standard upwind flux by a consistency term and a jump stabilization term. Finally, Burman and Stamm [11] proved that optimal convergence still holds also for quadratic and higher polynomial degrees when only the jump of the tangential part of the gradient is penalized.

In parallel to this development for hyperbolic problems, Continuous Interior Penalty (CIP) finite element methods were introduced in the 1970s by Babuška and Zlámal [2] for the biharmonic operator and by Douglas and Dupont [13] for second-order elliptic and parabolic problems. The idea behind CIP consists in penalizing the jump of the gradient of the discrete solution at interfaces between elements, thus weakly imposing $C^{1}$-continuity. More recently, CIP-methods experienced a further development. A priori error estimates that are uniform with respect to the diffusion coefficient have been obtained for CIP linear finite element approximations to

Date: October 31, 2007.

Key words and phrases. continuous and discontinuous Galerkin methods, hyperbolic problems, interior penalty. 
advection-diffusion equations by Burman and Hansbo [10]. A unified framework for the convergence analysis of both conforming and nonconforming linear finite elements with interior penalty (IP) has been proposed by Burman [7]. Finally, a CIP linear finite element method with a nonlinear shock-capturing term that rigorously guarantees a discrete maximum principle for advection-diffusion-reaction problems has been investigated by Burman and Ern [8].

In this paper we will show that the CIP-method for the transport equation can be seen as the asymptotic limit of the DG-method proposed in [5], provided the DG-formulation is augmented with the interior penalty term acting on the gradient jumps. Such a term was proposed as a stabilizing one for DG-methods in the approximation of elliptic problems by Romkes, Prudhomme and Oden [29] and by Brezzi, Cockburn, Marini and Süli [3] in a general framework focussing on stabilizing mechanisms for DG-methods. It does not downgrade the convergence order of the DG-method, rather it ensures more robustness with respect to variations in the stabilization parameter $\gamma_{0}$ acting on the solution jump. We prove that when $\gamma_{0}$ tends to infinity then the solution of the standard DG-method (without stabilization of the gradient jumps) converges to that of the unstabilized continuous Galerkin method. Two relevant properties follow. On the one hand a numerical flux can be defined for the continuous method as limit of the numerical flux of the discontinuous method as $\gamma_{0} \rightarrow \infty$ and, on the other hand, the DG-method as proposed in [5] is not stable if overstabilized (that is when $\gamma_{0}$ becomes too large) for advection dominated problems. A similar phenomenon was observed by Brezzi, Houston, Marini and Süli [4] for the subgrid viscosity method of Guermond [15] and is certainly true for the CIP using low order polynomials.

The asymptotic analysis is inspired by that for the elliptic case by Larson and Niklasson [22] and so is our discussion on the local fluxes in Section 5.

This paper is organized as follows. Section 2 introduces the two methods, the DGFEM and CGFEM, for the scalar hyperbolic equation. Special emphasis will be given to finding a uniform formalism for both methods. In Section 3 we recall $h$-convergence results for the continuous interior penalty method and for the augmented DG-method. In Section 4 we prove that the CIP-method can be considered as a limit of the DG-method if the jump stabilization parameter $\gamma_{0}$ tends to infinity. In Section 5 we discuss the local fluxes for the DG-method and the CG-method.

Some numerical examples for interior penalty stabilized finite element methods using continuous and discontinuous approximations are presented in Section 6, highlighting the theoretical results of Section 3 and 4 . Section 7 is left for the conclusions.

\section{Discontinuous and Continuous Finite Element Approximation with Interior PENALTy}

Let $\Omega$ be an open bounded and connected set in $\mathbb{R}^{d}, d=2,3$ with Lipschitz boundary $\partial \Omega$ and outer normal $n$. Moreover let $\beta \in\left[W^{1, \infty}(\Omega)\right]^{d}$ be a given vector field, $\mu \in L^{\infty}(\Omega)$ and $f \in L^{2}(\Omega)$ two given functions and $\partial \Omega^{ \pm}=\{x \in \partial \Omega$ : $\pm \beta(x) \cdot n(x)>0\}$ with $\partial \Omega^{+}$and $\partial \Omega^{-}$well separated. Consider the problem: find $u: \Omega \rightarrow \mathbb{R}$ such that

$$
\left\{\begin{aligned}
\mu u+\beta \cdot \nabla u & =f \text { in } \Omega \\
\left.u\right|_{\partial \Omega^{-}} & =0
\end{aligned}\right.
$$


Define $W=\left\{w \in L^{2}(\Omega): \beta \cdot \nabla w \in L^{2}(\Omega)\right\}$ and observe that functions in $W$ have traces in

$$
L^{2}(\partial \Omega ; \beta \cdot n)=\left\{v \in L^{2}(\partial \Omega): \int_{\partial \Omega}|\beta \cdot n| v^{2}<\infty\right\} .
$$

Consider the operator $A: W \ni w \mapsto \mu w+\beta \cdot \nabla w \in L^{2}(\Omega)$. Henceforth, it is assumed that there is $\mu_{0}>0$ such that

$$
\mu-\frac{1}{2} \nabla \cdot \beta \geq \mu_{0}, \quad \text { a.e. in } \Omega .
$$

Then, letting $V=\left\{w \in W:\left.w\right|_{\partial \Omega^{-}}=0\right\}, A: V \rightarrow L^{2}(\Omega)$ is an isomorphism, i.e., (1) is well-posed; see, e.g., [14, 27].

Let $\mathcal{K}$ be a finite element mesh of $\Omega$ into non-overlapping $d$-simplices. For $\kappa \in \mathcal{K}, h_{\kappa}$ denotes its diameter and set $h=\max _{\kappa \in \mathcal{K}} h_{\kappa}$. Assume that (i) $\mathcal{K}$ covers $\bar{\Omega}$ exactly, (ii) $\mathcal{K}$ does not contain any hanging nodes, and (iii) $\mathcal{K}$ is locally quasi-uniform in the sense that there exists a constant $\rho>0$, independent of $h$, such that

$$
\rho h_{\kappa} \leq \min _{\kappa^{\prime} \in \mathcal{N}(\kappa)} h_{\kappa^{\prime}},
$$

where $\mathcal{N}(\kappa)$ denotes the set of elements sharing at least one node with $\kappa$. Each $\kappa \in \mathcal{K}$ is an affine image of the unit simplex $\widehat{\kappa}$, i.e., $\kappa=F_{\kappa}(\widehat{\kappa})$. Let $\mathcal{F}_{\text {int }}$ denote the set of interior faces $((d-1)$-manifolds) of the mesh, i.e., the set of faces that are not included in the boundary $\partial \Omega$. The sets $\mathcal{F}_{ \pm}$denote the faces that are included in $\partial \Omega^{ \pm}$respectively and denote $\mathcal{F}=\mathcal{F}_{\text {int }} \cup \mathcal{F}_{+} \cup \mathcal{F}_{-}$. For $F \in \mathcal{F}, h_{F}$ denotes its diameter.

Let $p \geq 1$ and let $\mathbb{P}_{p}(\widehat{\kappa})$ be the space of polynomials of total degree $p$. Introduce the continuous and discontinuous finite element spaces

$$
\begin{aligned}
V_{h}^{p} & =\left\{v_{h} \in C^{0}(\bar{\Omega}): \forall \kappa \in \mathcal{K},\left.v_{h}\right|_{\kappa} \circ F_{\kappa} \in \mathbb{P}_{p}(\widehat{\kappa})\right\}, \\
W_{h}^{p} & =\left\{w_{h} \in L^{2}(\Omega): \forall \kappa \in \mathcal{K},\left.w_{h}\right|_{\kappa} \circ F_{\kappa} \in \mathbb{P}_{p}(\widehat{\kappa})\right\} .
\end{aligned}
$$

For a subset $R \subset \Omega,(\cdot, \cdot)_{R}$ denotes the $L^{2}(R)$-scalar product, $\|\cdot\|_{R}=(\cdot, \cdot)_{R}^{1 / 2}$ the associated norm, and $\|\cdot\|_{s, R}$ the $H^{s}(R)$-norm.

For $s \geq 1$, let $H^{s}(\mathcal{K})$ be the space of piecewise Sobolev $H^{s}$-functions. Let $\mathcal{S} \subset \mathcal{F}$ and define the scalar product $(\cdot, \cdot)_{\mathcal{S}}=\sum_{s \in \mathcal{S}}(\cdot, \cdot)_{s}$ and norm $\|\cdot\|_{\mathcal{S}}=(\cdot, \cdot)_{\mathcal{S}}^{1 / 2}$. For $v \in H^{2}(\mathcal{K})$ and an interior face $F=\kappa_{1} \cap \kappa_{2}$, where $\kappa_{1}$ and $\kappa_{2}$ are two distinct elements of $\mathcal{K}$ with respective outer normals $n_{1}$ and $n_{2}$, introduce the jump $[\nabla v]_{F}=$ $\left.\nabla v\right|_{\kappa_{1}} \cdot n_{1}+\left.\nabla v\right|_{\kappa_{2}} \cdot n_{2}$ (the subscript $F$ is dropped when there is no ambiguity). Similarly, for $v \in H^{1}(\mathcal{K})$, define the jump $[v]_{F}=\left.v\right|_{\kappa_{1}} n_{1}+\left.v\right|_{\kappa_{2}} n_{2}$. The average is defined for all functions $v \in H^{1}(\mathcal{K})$ by $\{v\}=\frac{1}{2}\left(\left.v\right|_{\kappa_{1}}+\left.v\right|_{\kappa_{2}}\right)$. On outer faces $F=\partial \kappa \cap \partial \Omega$ with outer normal $n$, the scalar-valued jump and the average are defined as $[v]_{F}=\left.v\right|_{\kappa} n$ resp. $\{v\}=\left.v\right|_{\kappa}$.

2.1. The discontinuous Galerkin approximation. On $W \times W$ define the discontinuous Galerkin bilinear form

$$
a(v, w)=((\mu-\nabla \cdot \beta) v, w)_{\Omega}-(v, \beta \cdot \nabla w)_{\Omega}+(\{\beta v\},[w])_{\mathcal{F}_{\text {int }} \cup \mathcal{F}_{+}},
$$

and on $H^{q}(\mathcal{K}) \times H^{q}(\mathcal{K}), q>\frac{3}{2}$, define the jump penalty and CIP bilinear form

$$
\begin{aligned}
& b_{0}(v, w)=\left(\beta_{n}[v],[w]\right)_{\mathcal{F}_{\text {int }}}, \\
& b_{1}(v, w)=\left(h_{F}^{2} \beta_{n}[\nabla v],[\nabla w]\right)_{\mathcal{F}_{\text {int }}}
\end{aligned}
$$


where $\left.\beta_{n}\right|_{F}=\|\beta \cdot n\|_{\infty, F}+\epsilon\|\beta \times n\|_{\infty, F}$, with $\epsilon \geq 0$ and where $\|\cdot\|_{\infty, F}$ denotes the $L^{\infty}$-norm on the face $F \in \mathcal{F}$. For the asymptotic analysis of Section 3.2 we assume that either $\epsilon>0$ or $\|\beta \cdot n\|_{\infty, F}>0$ for all faces $F$ of the mesh. Since $W^{1, \infty}(\Omega) \subset C^{0}(\bar{\Omega})$, the field $\beta$ is continuous by assumption and, therefore, the quantity $\beta_{n}$ is single-valued on all faces $F \in \mathcal{F}$.

The discontinuous finite element approximation of (1) consists of seeking $u_{d} \in W_{h}^{p}$ such that

$$
a\left(u_{d}, v_{d}\right)+\gamma_{0} b_{0}\left(u_{d}, v_{d}\right)+\gamma_{1} b_{1}\left(u_{d}, v_{d}\right)=\left(f, v_{d}\right)_{\Omega}, \quad \forall v_{d} \in W_{h}^{p}
$$

for $\gamma_{0}>0$ and $\gamma_{1} \geq 0$.

Remark 2.1. If the parameters $\epsilon$ and $\gamma_{1}$ are set equal to zero, then this method coincides with the one proposed in [5].

2.2. The continuous Galerkin approximation. The continuous finite element approximation with weakly imposed boundary condition is obtained by replacing the discontinuous finite element space $W_{h}^{p}$ by the continuous finite element space $V_{h}^{p}$. The problem becomes: find $u_{c} \in V_{h}^{p}$ such that

$$
a\left(u_{c}, v_{c}\right)+\gamma_{1} b_{1}\left(u_{c}, v_{c}\right)=\left(f, v_{c}\right)_{\Omega}, \quad \forall v_{c} \in V_{h}^{p} .
$$

Remark that the bilinear form $a(\cdot, \cdot)$, defined in $(5)$, simplifies to

$$
a(v, w)=((\mu-\nabla \cdot \beta) v, w)_{\Omega}-(v, \beta \cdot \nabla w)_{\Omega}+(\beta \cdot n v, w)_{\partial \Omega^{+}}
$$

and that $b_{0}\left(u_{c}, v_{c}\right)=0$ since $u_{c}$ is continuous.

2.3. Basic results. For $v \in H^{q}(\mathcal{K}), q>\frac{3}{2}$, consider the norm

$$
\|v\|^{2}=\left\|\mu_{0}^{\frac{1}{2}} v\right\|_{\Omega}^{2}+\frac{1}{2}\left\|\beta_{n}^{\frac{1}{2}} v\right\|_{\partial \Omega}^{2}+\gamma_{0} b_{0}(v, v)+\gamma_{1} b_{1}(v, v) .
$$

The well-posedness of the approximate problems, (8) and (9), results from the following lemma.

Lemma 2.2 (Coerciveness). For all $v \in H^{q}(\mathcal{K}), q>\frac{3}{2}$,

$$
a(v, v)+\gamma_{0} b_{0}(v, v)+\gamma_{1} b_{1}(v, v) \geq\|\| v \|^{2} .
$$

Proof. Straightforward verification using integration by parts and condition (2).

The next lemma shows the Galerkin orthogonality for both, the continuous and discontinuous, problems.

Lemma 2.3 (Consistency). Let $v \in V_{h}^{p}$ and $w \in W_{h}^{p}$ and assume $u \in H^{q}(\Omega)$, for $q>\frac{3}{2}$, then

$$
\begin{aligned}
a\left(u-u_{c}, v\right)+\gamma_{1} b_{1}\left(u-u_{c}, v\right) & =0, \\
a\left(u-u_{d}, w\right)+\gamma_{0} b_{0}\left(u-u_{d}, w\right)+\gamma_{1} b_{1}\left(u-u_{d}, w\right) & =0,
\end{aligned}
$$

where $u, u_{d}$ and $u_{c}$ denotes the solutions of (1), (8) resp. (9).

Proof. For the first equality, let $v \in V_{h}^{p}$ and observe that

$$
a\left(u_{c}, v\right)+\gamma_{1} b_{1}\left(u_{c}, v\right)=(f, v)_{\Omega}
$$


since $u_{c}$ is the solution of $(9)$. In addition note that

$$
\begin{aligned}
a(u, v) & =((\mu-\nabla \cdot \beta) u, v)_{\Omega}-(u, \beta \cdot \nabla v)_{\Omega}+(\beta \cdot n u, v)_{\mathcal{F}_{+}} \\
& =(\mu u+\beta \cdot \nabla u, v)_{\Omega}-(\beta \cdot n u, v)_{\partial \Omega}+(\beta \cdot n u, v)_{\mathcal{F}_{+}} \\
& =(\mu u+\beta \cdot \nabla u, v)_{\Omega}=(f, v)_{\Omega}
\end{aligned}
$$

having used integration by parts and the fact that $u_{\left.\right|_{\partial \Omega^{-}}}=0$. Moreover $b_{1}(u, v)=0$ and consequently (11) holds. For the second equality (12), let $w \in W_{h}^{p}$ and thus

$$
a\left(u_{d}, w\right)+\gamma_{0} b_{0}\left(u_{d}, w\right)+\gamma_{1} b_{1}\left(u_{d}, w\right)=(f, w)_{\Omega} .
$$

Finally, using integration by parts on each element, we have

$$
\begin{aligned}
a(u, w) & =((\mu-\nabla \cdot \beta) u, w)_{\Omega}-(u, \beta \cdot \nabla w)_{\Omega}+(\{\beta u\},[w])_{\mathcal{F}_{i n t} \cup \mathcal{F}_{+}} \\
& =(\mu u+\beta \cdot \nabla u, w)_{\Omega}-\sum_{\kappa \in \mathcal{K}}(\beta \cdot n u, w)_{\partial \kappa}+(\{\beta u\},[w])_{\mathcal{F}_{i n t} \cup \mathcal{F}_{+}} .
\end{aligned}
$$

Observe that

$$
\sum_{\kappa \in \mathcal{K}}(\beta \cdot n u, w)_{\partial \kappa}=(\{\beta u\},[w])_{\mathcal{F}}
$$

since $u$ is continuous. Therefore still using $u_{\left.\right|_{\partial \Omega^{-}}}=0$ we obtain

$$
a(u, w)=(\mu u+\beta \cdot \nabla u, w)_{\Omega}=(f, w)_{\Omega} .
$$

As above, since $u \in H^{q}(\Omega), q>\frac{3}{2}$,

$$
b_{0}(u, w)=0 \quad \text { and } \quad b_{1}(u, w)=0 .
$$

Thus, we have consistency in both cases.

The convergence analysis for the continuous and discontinuous method with weakly imposed boundary conditions and interior penalty gives the following result:

Theorem 2.4 (Convergence of CIP, [7]). Let $u \in H^{p+1}(\Omega), p \geq 1$, solve (1) and let $u_{c}$ solve (9). Then, there is a constant $c$, independent of $h$, such that

$$
\left\|u-u_{c}\right\| \mid \leq c h^{p+\frac{1}{2}}\|u\|_{p+1, \Omega} .
$$

Theorem 2.5 (Convergence of DGFEM, [5, 17]). Assume that $\gamma_{0}>0, \gamma_{1} \geq 0$, and that $u \in H^{p+1}(\Omega)$ with $p \geq 1$. Further assume that $\beta \in\left[W^{1, \infty}(\mathcal{K})\right]^{d}$. Then,

$$
\left\|u-u_{d}\right\| \mid \leq h^{p+\frac{1}{2}}\|u\|_{p+1, \Omega} .
$$

Remark 2.6. The proof of Theorem 2.5 in the case of $\gamma_{0}>0, \gamma_{1}=0$ is given in $[5,17]$. Adding the stabilization term $b_{1}(\cdot, \cdot)$ in their analysis is subject to some minor changes and yields optimal convergence.

Remark 2.7. For polynomial degrees $p \geq 2$ and $d=2$, stability of the discontinuous Galerkin method can also be obtained by penalizing only the jump of the tangential part of the gradient, for more details see [11].

Remark 2.8. Using a more involved analysis, but similar techniques, we may prove an inf-sup condition in a norm containing the $L^{2}$-norms of both the jumps of the discrete solution over element boundaries and the elementwise streamline derivative. 
2.4. Combining continuous and discontinuous finite element spaces. The above theory is not only limited to either continuous or discontinuous finite element spaces. Let $\left\{\Omega_{i}\right\}_{i=1}^{N}$ be a division of $\Omega$ into subregions $\Omega_{i}$, i.e. $\cup_{i=1}^{N} \overline{\Omega_{i}}=\bar{\Omega}$, and let $\mathcal{K}_{i}$ be a triangulation of $\Omega_{i}$. Then, define on $\Omega_{i}$ the continuous finite element space

$$
V_{h}^{p}\left(\Omega_{i}\right)=\left\{v_{h} \in C^{0}\left(\bar{\Omega}_{i}\right): \forall \kappa \in \mathcal{K}_{i},\left.v_{h}\right|_{\kappa} \circ F_{\kappa} \in \mathbb{P}_{p}(\widehat{\kappa})\right\}
$$

and match the subregions in a discontinuous manner

$$
W_{h, N}^{p}=\left\{v_{h} \in L^{2}(\Omega): \forall i=1, \ldots, N,\left.v_{h}\right|_{\Omega_{i}} \in V_{h}^{p}\left(\Omega_{i}\right)\right\} .
$$

Observe that the bilinear form $a(\cdot, \cdot)$, defined in (5), simplifies to

$$
a(v, w)=((\mu-\nabla \cdot \beta) v, w)_{\Omega}-(v, \beta \cdot \nabla w)_{\Omega}+(\{\beta v\},[w])_{\mathcal{F}_{i n t}^{N} \cup \mathcal{F}_{+}}
$$

for functions $v, w \in W_{h, N}^{p}$ and where

$$
\mathcal{F}_{\text {int }}^{N}=\left\{F \in \mathcal{F}_{\text {int }}: F \subset \partial \Omega_{j} \cap \partial \Omega_{k} \text { with } 1 \leq j, k \leq N, j \neq k\right\} .
$$

The stabilizing terms are then defined by

$$
\begin{aligned}
b_{0}(v, w) & =\left(\beta_{n}[v],[w]\right)_{\mathcal{F}_{\text {int }}^{N}}, \\
b_{1}(v, w) & =\left(h_{F}^{2} \beta_{n}[\nabla v],[\nabla w]\right)_{\mathcal{F}_{\text {int }} \backslash \mathcal{F}_{\text {int }}^{N}}+\delta\left(h_{F}^{2} \beta_{n}[\nabla v],[\nabla w]\right)_{\mathcal{F}_{\text {int }} \cap \mathcal{F}_{\text {int }}^{N}},
\end{aligned}
$$

with $\delta \geq 0$. A convergence analysis can be carried out combining the techniques of DG-methods and the CIP-method, see [12].

\section{The Continuous Galerkin Method as a limit of the Discontinuous Galerkin Method}

Hereafter the constant $c$ is considered a generic constant independent of $h$ and $\gamma_{0}$. Its actual value can change at each occurrence.

3.1. Preliminaries. We first recall an interpolation operator between discrete spaces $\mathcal{I}_{\mathrm{Os}}: W_{h}^{p} \rightarrow V_{h}^{p}$ endowed with a local interpolation property.

Let be $\kappa \in \mathcal{K}$. For a node $\nu$ in $\kappa$, set $\mathcal{K}_{\nu}=\left\{\kappa^{\prime} \in \mathcal{K} ; \nu \in \kappa^{\prime}\right\}$; then, for $w_{h} \in W_{h}^{p}$, define $\mathcal{I}_{\mathrm{Os}} w_{h}$ locally in $\kappa$ by the value it takes at all the Lagrangian nodes of $\kappa$ by setting

$$
\mathcal{I}_{\mathrm{Os}} w_{h}(\nu)=\left.\frac{1}{\operatorname{card}\left(\mathcal{K}_{\nu}\right)} \sum_{\kappa \in \mathcal{K}_{\nu}} w_{h}\right|_{\kappa}(\nu)
$$

Clearly, $\mathcal{I}_{\mathrm{Os}} w_{h} \in V_{h}^{p}$. The operator $\mathcal{I}_{\mathrm{Os}}$ is sometimes referred to as the Oswald interpolation operator; it has been considered in [7, 16, 21]. The next lemma points out some approximation results.

Lemma 3.1. There exists $c$, independent of $h_{\kappa}$ but not of the local mesh geometry, such that, for all $\kappa \in \mathcal{K}$, the following estimate holds:

$$
\begin{aligned}
\forall w_{h} \in W_{h}^{p}, \quad\left\|w_{h}-\mathcal{I}_{\mathrm{Os}} w_{h}\right\|_{\kappa} & \leq c h_{\kappa}^{\frac{1}{2}}\left\|\left[w_{h}\right]\right\|_{\mathcal{F}(\kappa)}, \\
\forall w_{h} \in W_{h}^{p}, \quad\left\|\nabla\left(w_{h}-\mathcal{I}_{\mathrm{Os}} w_{h}\right)\right\|_{\kappa} & \leq c h_{\kappa}^{-\frac{1}{2}}\left\|\left[w_{h}\right]\right\|_{\mathcal{F}(\kappa)},
\end{aligned}
$$

where $\mathcal{F}(\kappa)=\left\{F \in \mathcal{F}_{\text {int }}: F \cap \kappa \neq \emptyset\right\}$. 
3.2. Asymptotic limit $\gamma_{0} \rightarrow \infty$. Since we consider here consequences of an increasing $\gamma_{0}$, we may no longer use the triple norm defined in (10) since the parameter $\gamma_{0}$ is included in that definition. Instead a slightly modified norm is defined for this section

$$
\|v\|_{m}^{2}=\left\|\mu_{0}^{\frac{1}{2}} v\right\|_{\Omega}^{2}+\frac{1}{2}\left\|\beta_{n}^{\frac{1}{2}} v\right\|_{\partial \Omega}^{2}+b_{0}(v, v)+\gamma_{1} b_{1}(v, v) .
$$

Observe that if $v \in V_{h}^{p}$, then \|\|$v\|\mid=\| v \|_{m}$. One can easily show coercivity of the bilinear form $a(\cdot, \cdot)+b_{0}(\cdot, \cdot)+\gamma_{1} b_{1}(\cdot, \cdot)$ with respect to this norm as well.

Lemma 3.2 (Coerciveness). For all $v \in H^{q}(\mathcal{K}), q>\frac{3}{2}$,

$$
a(v, v)+b_{0}(v, v)+\gamma_{1} b_{1}(v, v) \geq\|v\|_{m}^{2} .
$$

Proof. The proof is similar to the one of Lemma 2.2.

Theorem 3.3. Let $u_{d}$ and $u_{c}$ be the solutions of the discontinuous resp. continuous problem (8) resp. (9). Let $u \in H^{p+1}(\Omega)$, with $p \geq 1$, solve (1). Then $u_{d}$ converges to $u_{c}$ as the parameter $\gamma_{0}$ tends to infinity provided the exact solution is sufficiently regular. Precisely, there exists a constant $c>0$, independent of $\gamma_{0}$ and $h$, such that

$$
\left\|u_{c}-u_{d}\right\|_{m} \leq \frac{c}{\gamma_{0}} h^{p-\frac{1}{2}}\|u\|_{p+1, \Omega}
$$

Proof. Let us denote $\eta=u_{c}-u_{d}$. Using coercivity, Lemma 3.2, and consistency leads to

$$
\begin{aligned}
\|\eta\|_{m}^{2} & \leq a(\eta, \eta)+b_{0}(\eta, \eta)+\gamma_{1} b_{1}(\eta, \eta) \\
& =a(\eta, \eta+v)+b_{0}(\eta, \eta+v)+\gamma_{1} b_{1}(\eta, \eta+v)
\end{aligned}
$$

for all $v \in V_{h}^{p}$. Indeed, subtracting (12) from (11) leads to

$$
a\left(u_{c}-u_{d}, v\right)+\gamma_{1} b_{1}\left(u_{c}-u_{d}, v\right)=0
$$

since $v$ is chosen to be continuous. For the same reason we have

$$
b_{0}\left(u_{c}-u_{d}, v\right)=0 .
$$

Define for simplicity

$$
\begin{array}{ll}
\mathcal{I}_{1}=((\mu-\nabla \cdot \beta) \eta, \eta+v)_{\Omega} & \mathcal{I}_{4}=(\{\beta \eta\},[\eta+v])_{\mathcal{F}_{+}} \\
\mathcal{I}_{2}=-(\eta, \beta \cdot \nabla(\eta+v))_{\Omega} & \mathcal{I}_{5}=b_{0}(\eta, \eta+v) \\
\mathcal{I}_{3}=(\{\beta \eta\},[\eta+v])_{\mathcal{F}_{i n t}} & \mathcal{I}_{6}=\gamma_{1} b_{1}(\eta, \eta+v)
\end{array}
$$

Hence $\|\eta\|_{m}^{2} \leq \sum_{i=1}^{6} \mathcal{I}_{i}$. Set $v=-\mathcal{I}_{\text {Os }} \eta \in V_{h}^{p}$. For the first four terms the Cauchy-Schwarz inequality and Lemma 3.1 are used:

$$
\begin{aligned}
\mathcal{I}_{1} & \leq c\left\|\mu_{0}^{\frac{1}{2}} \eta\right\|_{\Omega}\left\|\eta-\mathcal{I}_{\mathrm{Os}} \eta\right\|_{\Omega} \leq c h^{\frac{1}{2}}\|\eta \eta\|_{m}\|[\eta]\|_{\mathcal{F}_{i n t}} \\
\mathcal{I}_{2} & \leq\|\eta\|_{\Omega}\left\|\beta \cdot \nabla\left(\eta-\mathcal{I}_{\mathrm{Os}} \eta\right)\right\|_{\Omega} \leq c h^{-\frac{1}{2}}\left\|\mu_{0}^{\frac{1}{2}} \eta\right\|_{\Omega}\|[\eta]\|_{\mathcal{F}_{i n t}} \leq c h^{-\frac{1}{2}}\|\eta \eta\|_{m}\|[\eta]\|_{\mathcal{F}_{\text {int }}} \\
\mathcal{I}_{3} & =\frac{1}{2} \sum_{\kappa \in \mathcal{K}}\left(\beta \eta,\left[\eta-\mathcal{I}_{\mathrm{Os}} \eta\right]\right)_{\partial \kappa \backslash \partial \Omega} \leq c h^{-\frac{1}{2}} \sum_{\kappa \in \mathcal{K}}\|\eta\|_{\kappa}\|[\eta]\|_{\partial \kappa \backslash \partial \Omega} \\
& \leq c h^{-\frac{1}{2}}\|\eta\|_{\Omega}\|[\eta]\|_{\mathcal{F}_{i n t}} \leq c h^{-\frac{1}{2}}\|\eta\|\left\|_{m}\right\|[\eta] \|_{\mathcal{F}_{i n t}} \\
\mathcal{I}_{4} & =\sum_{\kappa \in \mathcal{K}}\left(\beta \cdot n \eta, \eta-\mathcal{I}_{\mathrm{Os}} \eta\right)_{\partial \kappa \cap \partial \Omega^{+}} \leq c h^{-\frac{1}{2}} \sum_{\kappa \in \mathcal{K}}\left\||\beta \cdot n|^{\frac{1}{2}} \eta\right\|_{\partial \kappa \cap \partial \Omega^{+}}\left\|\eta-\mathcal{I}_{\text {Os }} \eta\right\|_{\kappa} \\
\leq & c\|\eta\|\left\|_{m}\right\|[\eta] \|_{\mathcal{F}_{i n t}}
\end{aligned}
$$


where for the third term, the trace inequality and the fact that $\mathcal{I}_{\mathrm{Os}} \eta \in V_{h}^{p}$ is used. In a similar fashion we obtain

$$
\mathcal{I}_{5}=\left\|\beta_{n}^{\frac{1}{2}}[\eta]\right\|_{\mathcal{F}_{\text {int }}}^{2} \leq c\|\| \eta\|\|_{m}\|[\eta]\|_{\mathcal{F}_{\text {int }}} .
$$

For $\mathcal{I}_{6}$ the trace inequality and Lemma 3.1 is used:

$$
\begin{aligned}
\mathcal{I}_{6} & \leq c\|\beta\|_{\infty, \Omega}\|h[\nabla \eta]\|_{\mathcal{F}_{\text {int }}}\left\|h\left[\nabla\left(\eta-\mathcal{I}_{\mathrm{Os}} \eta\right)\right]\right\|_{\mathcal{F}_{\text {int }}} \\
& \leq c b_{1}(\eta, \eta)^{\frac{1}{2}} h^{\frac{1}{2}} \sum_{\kappa \in \mathcal{K}}\left\|\nabla\left(\eta-\mathcal{I}_{\mathrm{Os}} \eta\right)\right\|_{\kappa} \leq c\|\eta\|\left\|_{m}\right\|[\eta] \|_{\mathcal{F}_{\text {int }} .} .
\end{aligned}
$$

Respecting all six bounds yields

$$
\|\eta \eta\|_{m} \leq c\left(h^{\frac{1}{2}}+2 h^{-\frac{1}{2}}+3\right)\|[\eta]\|_{\mathcal{F}_{i n t}} \leq c h^{-\frac{1}{2}}\|[\eta]\|_{\mathcal{F}_{\text {int }}},
$$

since $h<1$. Then, using coercivity, Lemma 2.2, and consistency leads to

$$
\begin{aligned}
\gamma_{0}\|[\eta]\|_{\mathcal{F}_{\text {int }}}^{2} & \leq a(\eta, \eta)+\gamma_{0} b_{0}(\eta, \eta)+\gamma_{1} b_{1}(\eta, \eta) \\
& =a\left(u_{c}-u, \eta\right)+\gamma_{0} b_{0}\left(u_{c}-u, \eta\right)+\gamma_{1} b_{1}\left(u_{c}-u, \eta\right) \\
& =a\left(u_{c}-u, \eta\right)+\gamma_{1} b_{1}\left(u_{c}-u, \eta\right) \\
& =a\left(u_{c}-u, \eta-\mathcal{I}_{\text {Os }} \eta\right)+\gamma_{1} b_{1}\left(u_{c}-u, \eta-\mathcal{I}_{\text {Os }} \eta\right)
\end{aligned}
$$

since $u_{c}-u$ is continuous. Using analogous arguments as for bounding $\|\eta\|_{m}$, we conclude that

$$
\gamma_{0}\|[\eta]\|_{\mathcal{F}_{\text {int }}}^{2} \leq c\left(h^{\frac{1}{2}}+2 h^{-\frac{1}{2}}+2\right) \mid\left\|u_{c}-u\right\|\|\|[\eta] \|_{\mathcal{F}_{\text {int }}}
$$

and hence

$$
\|[\eta]\|_{\mathcal{F}_{\text {int }}} \leq \frac{c}{\gamma_{0}} h^{-\frac{1}{2}}\left\|u_{c}-u\right\| \| .
$$

The convergence of the continuous approximation, proposition 2.4, leads to the bound

$$
\|[\eta]\|_{\mathcal{F}_{\text {int }}} \leq \frac{c}{\gamma_{0}} h^{p}\|u\|_{p+1, \Omega} .
$$

Combining (17) and (18) yields

$$
\|\eta\|\left\|_{m} \leq \frac{c}{\gamma_{0}} h^{p-\frac{1}{2}}\right\| u \|_{p+1, \Omega}
$$

\section{Local Flux Conservation}

In this section, we will study the behavior of the numerical flux of the DG-method in the asymptotic limit and show how this may be used to define a conservative numerical flux also for the continuous Galerkin method [18, 22].

Consider problem (1) with $\mu=0$ and $\nabla \cdot \beta=0$, i.e. the pure transport problem, and let $\Lambda \subset \Omega$ be a subdomain of $\Omega$. We associate to $\Lambda$ its outer normal $n_{\Lambda}$. Further denote $\chi_{\Lambda}$ the characteristic function on $\Lambda$ defined by $\chi_{\Lambda}=1$ on $\Lambda$ and $\chi_{\Lambda}=0$ on $\Omega \backslash \Lambda$. Multiplying the first line of (1) by $\chi_{\Lambda}$ and integrating by parts on $\Lambda$ yields

$$
\int_{\partial \Lambda} \sigma_{\Lambda}(u)=\int_{\Lambda} f
$$


since $\beta$ is divergence free, $\left.\nabla \chi_{\kappa}\right|_{\kappa}=0$ and where $\sigma_{\Lambda}(u)=\beta \cdot n_{\Lambda} u$ denotes the problem flux. For the discontinuous Galerkin method the same relation is true on each element $\kappa$ and for a numerical flux $\Sigma_{\kappa, \gamma_{0}}^{d}$ defined by

$$
\Sigma_{\kappa, \gamma_{0}}^{d}(w)= \begin{cases}\sigma_{\kappa}(\{w\})+\gamma_{0} \beta_{n}[w] & \text { on } \mathcal{F}_{\text {int }} \cap \partial \kappa \\ \sigma_{\kappa}(w) & \text { on } \mathcal{F}_{+} \cap \partial \kappa \\ 0 & \text { on } \mathcal{F}_{-} \cap \partial \kappa\end{cases}
$$

for all $w \in W_{h}^{p}$. Then, replacing the test function in (8) by the characteristic function $\chi_{\kappa}$ where $\kappa \in \mathcal{K}$, leads to

$$
\int_{\partial \kappa} \Sigma_{\kappa, \gamma_{0}}^{d}\left(u_{d}\right)=\int_{\kappa} f
$$

Hence there is a local flux conservation for the discontinuous Galerkin method. Now since the continuous Galerkin method can be considered as the limit of the discontinuous Galerkin method, we define a numerical flux for the continuous Galerkin method by

$$
\Sigma_{\kappa}^{c}\left(u_{c}\right)= \begin{cases}\sigma_{\kappa}\left(u_{c}\right)+\beta_{n}[\rho] & \text { on } \mathcal{F}_{\text {int }} \cap \partial \kappa \\ \sigma_{\kappa}\left(u_{c}\right) & \text { on } \mathcal{F}_{+} \cap \partial \kappa \\ 0 & \text { on } \mathcal{F}_{-} \cap \partial \kappa\end{cases}
$$

where $\rho$ is defined by the problem: find $\rho \in W_{h}^{p} \backslash V_{h}^{p}$ such that

$$
b_{0}(\rho, w)=(f, w)_{\Omega}-a\left(u_{c}, w\right)-\gamma_{1} b_{1}\left(u_{c}, w\right) \quad \forall w \in W_{h}^{p} .
$$

Lemma 4.1. The problem (21) is well posed.

Proof. Consider the following auxiliary problem: find $\rho \in W_{h}^{p} \backslash V_{h}^{p}$ such that

$$
b_{0}(\rho, \bar{w})=(f, \bar{w})_{\Omega}-a\left(u_{c}, \bar{w}\right)-\gamma_{1} b_{1}\left(u_{c}, \bar{w}\right) \quad \forall \bar{w} \in W_{h}^{p} \backslash V_{h}^{p} .
$$

Since the trial and test space are equal and since the kernel of $b_{0}(\cdot, \cdot)$ in $W_{h}^{p} \backslash V_{h}^{p}$ is zero, one can apply the standard theory to show the well posedness. Remember that we assume that either $\varepsilon>0$ in the definition of $\beta_{n}$ or $\|\beta \cdot n\|_{\infty, F}>0$ on all faces of the mesh. Then, observe that one can decompose every function $w \in W_{h}^{p}$ in $w=\bar{w}+v$ with $\bar{w} \in W_{h}^{p} \backslash V_{h}^{p}$ and $v \in V_{h}^{p}$. Then

$$
b_{0}(\rho, v)=0 \quad \text { and } \quad(f, v)_{\Omega}-a\left(u_{c}, v\right)-\gamma_{1} b_{1}\left(u_{c}, v\right)=0,
$$

owing to the fact that $v$ is continuous and to the consistency of the continuous Galerkin method. Therefore this auxiliary problem is equivalent to the original one and this implies the uniqueness of $\rho$.

Lemma 4.2. Let $u_{d}=u_{d}\left(\gamma_{0}\right)$ be the solution of (8) corresponding to a given value of $\gamma_{0}$; then

$$
\lim _{\gamma_{0} \rightarrow \infty} \gamma_{0} b_{0}\left(u_{d}, w\right)=b_{0}(\rho, w)
$$

for all $w \in W_{h}^{p} \backslash V_{h}^{p}$.

Proof. Using the discrete formulation of the discontinuous Galerkin method and the fact that the bilinear forms $a(\cdot, \cdot)$ and $b_{1}(\cdot, \cdot)$ are continuous with respect to both variables yields

$$
\begin{aligned}
\lim _{\gamma_{0} \rightarrow \infty} \gamma_{0} b_{0}\left(u_{d}, w\right) & =(f, w)_{\Omega}-\lim _{\gamma_{0} \rightarrow \infty} a\left(u_{d}, w\right)-\lim _{\gamma_{0} \rightarrow \infty} \gamma_{1} b_{1}\left(u_{d}, w\right) \\
& =(f, w)-a\left(u_{c}, w\right)-\gamma_{1} b_{1}\left(u_{c}, w\right)=b_{0}(\rho, w)
\end{aligned}
$$

for all $w \in W_{h}^{p} \backslash V_{h}^{p}$ since $\left\|u_{d}-u_{c}\right\|_{\Omega} \rightarrow 0$ as $\gamma_{0} \rightarrow \infty$. 
Proposition 4.3. Let $F \in \mathcal{F}$ be an arbitrary face of an arbitrary element $\kappa \in \mathcal{K}$. Then the numerical flux $\Sigma_{\kappa, \gamma_{0}}^{d}\left(u_{d}\right)$ converges to $\Sigma_{\kappa}^{c}\left(u_{c}\right)$, i.e.

$$
\lim _{\gamma_{0} \rightarrow \infty}\left(\Sigma_{\kappa, \gamma_{0}}^{d}\left(u_{d}\right),[w]\right)_{F}=\left(\Sigma_{\kappa}^{c}\left(u_{c}\right),[w]\right)_{F}
$$

for all $w \in W_{h}^{p} \backslash V_{h}^{p}$.

Proof. On faces contained in $\mathcal{F}_{-}$the limit is obvious since both fluxes are zero. Since the exact flux $\sigma_{\kappa}(\cdot)$ is continuous we have on faces contained in $\mathcal{F}_{+}$that $\sigma_{\kappa}\left(\left\{u_{d}\right\}\right) \rightarrow \sigma_{\kappa}\left(\left\{u_{c}\right\}\right)=\sigma_{\kappa}\left(u_{c}\right)$. On interior faces we use the same argument and Lemma 4.2 .

Corollary 4.4. For the continuous Galerkin method, we still have the local conservation property, i.e.

$$
\int_{\partial \kappa} \Sigma_{\kappa}^{c}\left(u_{c}\right)=\int_{\kappa} f
$$

Proof. Choose $w=\chi_{\kappa}$ in Proposition 4.3 and sum over all faces of $\kappa$.

4.1. Behavior of the numerical flux as $h \rightarrow 0$. Since all fluxes are equal on the boundary of an element, i.e.

$$
\int_{\partial \kappa} \Sigma_{\kappa}^{c}\left(u_{c}\right)=\int_{\partial \kappa} \Sigma_{\kappa, \gamma_{0}}^{d}\left(u_{d}\right)=\int_{\partial \kappa} \sigma_{\kappa}(u)=\int_{\kappa} f
$$

it is evident that

$$
\int_{\partial \kappa}\left(\sigma_{\kappa}(u)-\Sigma_{\kappa}^{c}\left(u_{c}\right)\right)=0 \quad \text { and } \quad \int_{\partial \kappa}\left(\sigma_{\kappa}(u)-\Sigma_{\kappa, \gamma_{0}}^{d}\left(u_{d}\right)\right)=0
$$

for all $h>0$. For what concerns the exact flux of the difference of the solutions consider the following two lemmas.

Lemma 4.5. The difference of the flux of the exact solution $u$ and the flux of the numerical solution $u_{d}$ converges to zero as $h \rightarrow 0$ with a convergence rate of $p$, i.e.

$$
\sum_{\kappa \in \mathcal{K}}\left|\int_{\partial \kappa}\left(\sigma_{\kappa}(u)-\sigma_{\kappa}\left(u_{d}\right)\right)\right| \leq c h^{p}\|u\|_{p+1, \Omega}
$$

Proof. Applying equality (22) and the Cauchy-Schwarz inequality yields

$$
\begin{gathered}
\sum_{\kappa \in \mathcal{K}}\left|\int_{\partial \kappa}\left(\sigma_{\kappa}(u)-\sigma_{\kappa}\left(u_{d}\right)\right)\right|=\sum_{\kappa \in \mathcal{K}}\left|\int_{\partial \kappa}\left(\Sigma_{\kappa, \gamma_{0}}^{d}\left(u_{d}\right)-\sigma_{\kappa}\left(u_{d}\right)\right)\right| \\
\leq\left(\sum_{\kappa \in \mathcal{K}} h_{\kappa}^{-1}\left\|\Sigma_{\kappa, \gamma_{0}}^{d}\left(u_{d}\right)-\sigma_{\kappa}\left(u_{d}\right)\right\|_{\partial \kappa}^{2}\right)^{\frac{1}{2}}\left(\sum_{\kappa \in \mathcal{K}} \int_{\partial \kappa} h_{\kappa}\right)^{\frac{1}{2}} .
\end{gathered}
$$

Observe that $\sum_{\kappa \in \mathcal{K}} \int_{\partial \kappa} h_{\kappa} \leq c$ using the shape regularity of the mesh. By the definition of the numerical flux $\Sigma_{\kappa, \gamma_{0}}^{d}$, the error estimate of Theorem 2.5 and since

$$
\left.\beta \cdot n_{\kappa}\left(\left\{u_{d}\right\}-u_{d}\right)\right|_{\partial \kappa}=-\left.\frac{1}{2} \beta \cdot\left[u_{d}\right]\right|_{\partial \kappa} \quad \forall \kappa \in \mathcal{K}
$$

we get

$$
\begin{aligned}
\sum_{\kappa \in \mathcal{K}}\left|\int_{\partial \kappa}\left(\sigma_{\kappa}(u)-\sigma_{\kappa}\left(u_{d}\right)\right)\right| & \leq c\left(\sum_{\kappa \in \mathcal{K}}\left\|h_{\kappa}^{-\frac{1}{2}} \beta_{n}^{\frac{1}{2}} u_{d}\right\|_{\partial \kappa \cap \partial \Omega^{-}}^{2}+\sum_{\kappa \in \mathcal{K}}\left\|h_{\kappa}^{-\frac{1}{2}} \beta_{n}^{\frac{1}{2}}\left[u_{d}\right]\right\|_{\partial \kappa \backslash \partial \Omega}^{2}\right)^{\frac{1}{2}} \\
& \leq c\left\|h^{-\frac{1}{2}}\left(u-u_{d}\right)\right\| \leq c h^{p}\|u\|_{p+1, \Omega} .
\end{aligned}
$$


For the next lemma assume for simplicity that $\beta \in \mathbb{R}^{2}$ and $\mu \in \mathbb{R}$.

Lemma 4.6. Assume that $f \in H^{p}(\Omega)$ and that the mesh is globally quasi-uniform. The difference of the flux of the exact solution $u$ and the flux of the numerical solution $u_{c}$ converges to zero as $h \rightarrow 0$ with a convergence rate of $p$, i.e.

$$
\sum_{\kappa \in \mathcal{K}}\left|\int_{\partial \kappa}\left(\sigma_{\kappa}(u)-\sigma_{\kappa}\left(u_{c}\right)\right)\right| \leq c h^{p}\left(\|f\|_{p, \Omega}+\|u\|_{p+1, \Omega}\right) .
$$

Proof. Applying again equality (22) and the Cauchy-Schwarz inequality yields

$$
\begin{array}{r}
\sum_{\kappa \in \mathcal{K}}\left|\int_{\partial \kappa}\left(\sigma_{\kappa}(u)-\sigma_{\kappa}\left(u_{c}\right)\right)\right|=\sum_{\kappa \in \mathcal{K}}\left|\int_{\partial \kappa}\left(\Sigma_{\kappa}^{c}\left(u_{c}\right)-\sigma_{\kappa}\left(u_{c}\right)\right)\right| \\
\leq\left(\sum_{\kappa \in \mathcal{K}} h_{\kappa}^{-1}\left\|\Sigma_{\kappa}^{c}\left(u_{c}\right)-\sigma_{\kappa}\left(u_{c}\right)\right\|_{\partial \kappa}^{2}\right)^{\frac{1}{2}}\left(\sum_{\kappa \in \mathcal{K}} \int_{\partial \kappa} h_{\kappa}\right)^{\frac{1}{2}} .
\end{array}
$$

Observe that $\sum_{\kappa \in \mathcal{K}} \int_{\partial \kappa} h_{\kappa} \leq c$ using the shape regularity of the mesh. Using the definition of the numerical flux $\Sigma_{\kappa}^{c}$ yields

$$
\begin{aligned}
\sum_{\kappa \in \mathcal{K}}\left|\int_{\partial \kappa}\left(\sigma_{\kappa}(u)-\sigma_{\kappa}\left(u_{c}\right)\right)\right| & \leq c\left(\sum_{\kappa \in \mathcal{K}}\left\|h_{\kappa}^{-\frac{1}{2}} \beta_{n}^{\frac{1}{2}} u_{c}\right\|_{\partial \kappa \cap \partial \Omega^{-}}^{2}+\sum_{\kappa \in \mathcal{K}}\left\|h_{\kappa}^{-\frac{1}{2}} \beta_{n}^{\frac{1}{2}}[\rho]\right\|_{\partial \kappa \backslash \partial \Omega}^{2}\right)^{\frac{1}{2}} \\
& \left.\leq c(23) \quad c h^{2 p}\|u\|_{p+1, \Omega}^{2}+h^{-1} b_{0}(\rho, \rho)\right)^{\frac{1}{2}} .
\end{aligned}
$$

Now, let $\pi_{h} \rho \in V_{h}^{p}$ denote the $L^{2}$-projection of $\rho$ onto the continuous space. By equations (14) and (15) it follows that

$$
\left\|\rho-\pi_{h} \rho\right\|_{\Omega}^{2}+h\left\|\rho-\pi_{h} \rho\right\|_{\partial \Omega^{-}}^{2}+\gamma_{1} h b_{1}\left(\rho-\pi_{h} \rho, \rho-\pi_{h} \rho\right) \leq \operatorname{ch} b_{0}(\rho, \rho) .
$$

On the other hand using integration by parts we have that

$$
a\left(u_{c}, \rho\right)=\left(\mu u_{c}+\beta \cdot \nabla u_{c}, \rho\right)_{\Omega}-\left(\beta \cdot n u_{c}, \rho\right)_{\partial \Omega^{-}} .
$$

By the definition of $\rho$, the Galerkin orthogonality and the orthogonality of the $L^{2}$-projection we deduce that

$$
\begin{aligned}
& b_{0}(\rho, \rho) \\
&=h^{-1}\left((f, \rho)_{\Omega}-a\left(u_{c}, \rho\right)-\gamma_{1} b_{1}\left(u_{c}, \rho\right)\right) \\
&=\left(\left(f-\pi_{h} f, \rho-\pi_{h} \rho\right)_{\Omega}-\left(\beta \cdot \nabla u_{c}-\mathcal{I}_{\mathrm{Os}}\left(\beta \cdot \nabla u_{c}\right), \rho-\pi_{h} \rho\right)_{\Omega}\right. \\
&\left.+\left(\beta \cdot n u_{c}, \rho-\pi_{h} \rho\right)_{\partial \Omega^{-}}-\gamma_{1} b_{1}\left(u_{c}, \rho-\pi_{h} \rho\right)\right)^{\frac{1}{2}} \\
& \leq c\left(\left\|f-\pi_{h} f\right\|_{\Omega}+\left\|\beta \cdot \nabla u_{c}-\mathcal{I}_{\mathrm{Os}}\left(\beta \cdot \nabla u_{c}\right)\right\|_{\Omega}+h^{-\frac{1}{2}}\left\|\beta_{n}^{\frac{1}{2}} u_{c}\right\|_{\partial \Omega^{-}}+\gamma_{1}^{\frac{1}{2}} h^{-\frac{1}{2}} b_{1}\left(u_{c}, u_{c}\right)^{\frac{1}{2}}\right) \\
&\left(\left\|\rho-\pi_{h} \rho\right\|_{\Omega}+h^{\frac{1}{2}}\left\|\rho-\pi_{h} \rho\right\|_{\partial \Omega^{-}}+\gamma_{1}^{\frac{1}{2}} h^{\frac{1}{2}} b_{1}\left(\rho-\pi_{h} \rho, \rho-\pi_{h} \rho\right)^{\frac{1}{2}}\right) .
\end{aligned}
$$

The inequality (24) leads to the following bound for

$$
\begin{aligned}
b_{0}(\rho, \rho) & \leq h^{\frac{1}{2}} b_{0}(\rho, \rho)^{\frac{1}{2}}\left(h^{p}\|f\|_{p, \Omega}+\left(1+\gamma_{1}^{\frac{1}{2}}\right) h^{-\frac{1}{2}} b_{1}\left(u_{c}, u_{c}\right)^{\frac{1}{2}}+h^{-\frac{1}{2}}\left\|\beta_{n}^{\frac{1}{2}} u_{c}\right\|_{\partial \Omega^{-}}\right) \\
& \leq h^{\frac{1}{2}} b_{0}(\rho, \rho)^{\frac{1}{2}}\left(h^{p}\|f\|_{p, \Omega}+h^{-\frac{1}{2}}\left\|u-u_{c}\right\|\right)
\end{aligned}
$$

and therefore

$$
h^{-\frac{1}{2}} b_{0}(\rho, \rho)^{\frac{1}{2}} \leq h^{p}\left(\|f\|_{p, \Omega}+\|u\|_{p+1, \Omega}\right)
$$




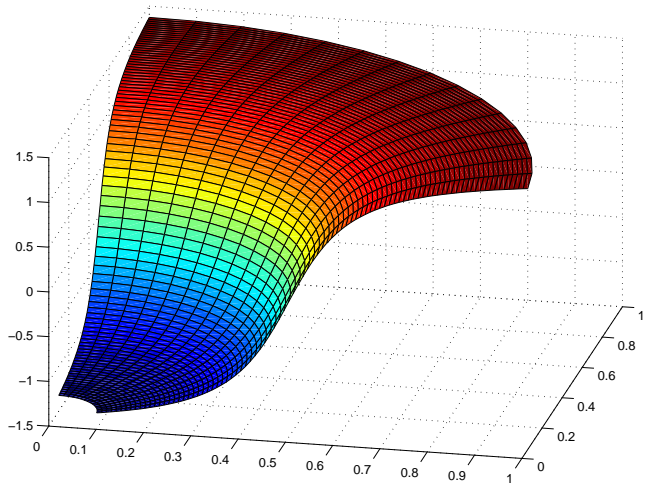

Figure 1. The exact solution.

by the estimate of Theorem 2.4. Inserting (25) into (23) leads to the desired result.

\section{Numerical Results}

The following transport problem is considered. Let $\Omega \subset \mathbb{R}^{2}$ be the domain defined by $\Omega=\left\{(x, y) \in \mathbb{R}^{+} \times \mathbb{R}^{+}: 0.1 \leq \sqrt{x^{2}+y^{2}} \leq 1\right\}$. The problem consists of seeking $u$ such that

$$
\left\{\begin{aligned}
\mu u+\beta \cdot \nabla u & =0 \text { in } \Omega, \\
\left.u\right|_{\partial \Omega^{-}} & =g(y)
\end{aligned}\right.
$$

where

$$
\beta(x, y)=\left(\begin{array}{c}
y \\
-x
\end{array}\right) \frac{1}{\sqrt{x^{2}+y^{2}}} \text { and } g(y)=\arctan \left(\frac{y-0.5}{0.1}\right) .
$$

Then, the solution writes

$$
u(x, y)=e^{\mu \sqrt{x^{2}+y^{2}} \arcsin \left(\frac{y}{\sqrt{x^{2}+y^{2}}}\right)} \arctan \left(\frac{\sqrt{x^{2}+y^{2}}-0.5}{0.1}\right) .
$$

The reaction coefficient $\mu=0.01$ is chosen sufficiently small such that the transport is dominating the reaction. Figure 1 shows the exact solution $u$. We consider sequences of unstructured triangular meshes for polynomial degrees $p=1, \ldots, 5$. For the computations the $\mathrm{C}++$ library life, a unified $\mathrm{C}++$ implementation of the finite and spectral element methods in $1 \mathrm{D}, 2 \mathrm{D}$ and $3 \mathrm{D}$, is used, see $[25,26]$.

5.1. Optimal choice of the stabilization parameter of continuous interior penalty method. For the continuous interior penalty method on rectangular meshes the optimal choice of the stabilization parameter $\gamma_{1}$ with respect to the polynomial degree is carried out yielding that $\gamma_{1} \sim p^{-3.5}$, see [9] for more details. Figure 2 shows the $L^{2}$-error depending of $\gamma_{1}$ for a fixed triangular mesh with size $h=0.05$ and for each polynomial degree. The optimal choice for this example is illustrated in the following table: 


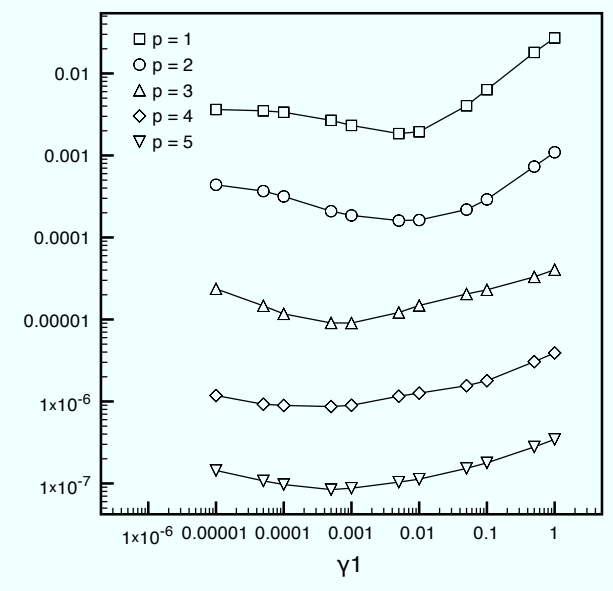

FIGURE 2. Behavior of the error of the continuous interior penalty method with respect to the stabilization parameter $\gamma_{1}$ for different polynomial degrees and fixed $h$ measured in the $L^{2}{ }^{2}$ norm.

\begin{tabular}{|c|c|c|c|c|c|}
\hline$p$ & 1 & 2 & 3 & 4 & 5 \\
\hline$\gamma_{1, \text { opt }}$ & 0.005 & 0.005 & 0.001 & 0.0005 & 0.0005 \\
\hline
\end{tabular}

These values will be the reference values for the following computations.

5.2. Convergence with respect to $h$ and $p$. Since $u \in C^{\infty}(\Omega), u \in H^{r}(\Omega)$ for all $r \geq 0$. Hence the solution of continuous method satisfies

$$
\left\|u-u_{c}\right\| \leq \operatorname{ch}^{p+\frac{1}{2}}\|u\|_{p+1, \Omega} .
$$

Similarly, for the discontinuous method, we get

$$
\left\|u-u_{d}\right\| \mid \leq \operatorname{ch}^{p+\frac{1}{2}}\|u\|_{p+1, \Omega} .
$$

Observe that the $L^{2}{ }^{-}$norm is controlled by the triple norm, i.e. $\|v\|_{\Omega} \leq\|\| v \|$. Note that the $h p$-analysis carried out in [9] for the continuous interior penalty method and in [17] for the DG-method only hold on rectangular meshes, whereas an $h$ analysis can be carried out for any polynomial degree $p$ on triangular meshes for both methods. Figure 3 shows the $L^{2}$-norm of the error of the upwind discontinuous method, i.e. $\gamma_{0}=0.5, \gamma_{1}=0$, in dashed line and the continuous interior penalty method with optimal stabilization parameter $\gamma_{1}$ according to section 5.1 in solid line.

Observe the optimal convergence with respect to $h$ and the exponential convergence with respect to $p$.

5.3. CG-method as limit of the DG-method. Here we test the case when the stabilization parameter $\gamma_{0}$ of the DG-method increases to infinity for fixed mesh size $h=0.05$. The theoretical result tells us that the $L^{2}{ }^{2}$ norm of the difference between the solutions of the discontinuous and continuous methods converges to zero. The order of convergence is predicted as one. That is exactly what can be observed for a sufficiently large $\gamma_{0}$ in figure 4 (a) for both cases $\gamma_{1}=0$ (solid line) 


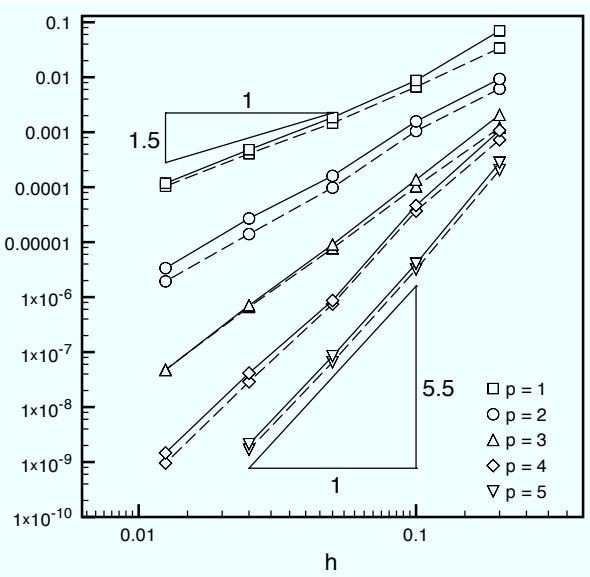

(a)

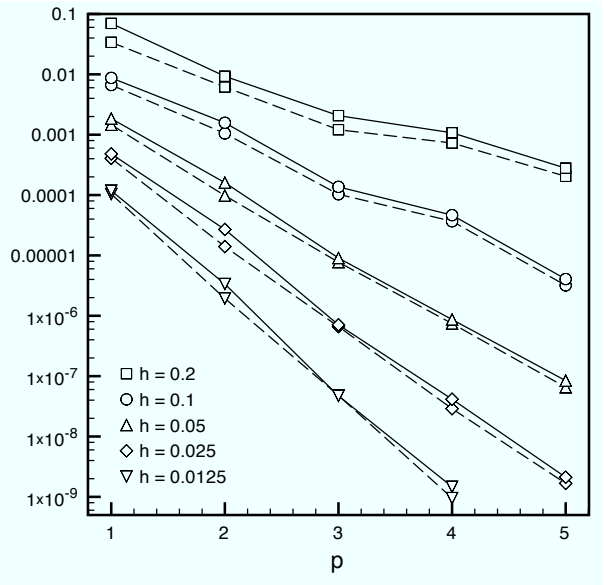

(b)

FiguRE 3. Convergence behavior with respect to $h(a)$ and $p$ (b) of the upwind discontinuous method ( $\gamma_{0}=0.5, \gamma_{1}=0$, dashed line) and the continuous interior penalty method with optimal parameter $\gamma_{1}$ (solid line).

and $\gamma_{1}>0$ (dashed line). The parameter $\gamma_{1}>0$ is chosen according to the optimal criterion for the continuous method as illustrated in section 5.1.

Figure 4(b) shows the $L^{2}$-norm of the difference between the exact solution $u$ and the DG-approximation when $\gamma_{0}$ tends to infinity. We see that the parameter $\gamma_{0}=0.5$ corresponding to upwind stabilisation is a good choice for all polynomial orders. Although it does not always correspond to the optimal choice for the error in the $L^{2}$-norm the difference is very small.

Figure 4(b) also shows that for a fixed $h$ there exists a $\gamma_{0}$ such that the DGmethod is more precise than the continuous method without interior penalty. On the other hand if the DG-method is augmented with the gradient jump stabilization the solution is robust to overstabilization (that is when $\gamma_{0}$ becomes too large), especially for high order approximations. Finally the results reported in Figure 3 and 4(b) show that the CIP-method yields similar accuracy as the upwind DGmethod. Indeed, in this numerical example, the CIP-method with the optimal parameter $\gamma_{1}$ leads to an approximation with an accuracy very similar to that of the upwind DG-method, but using much fewer degrees of freedom.

\section{Conclusions}

In this paper we have compared theoretically and numerically two methods which are suitable for the approximation of transport dominated advection-reaction problems: the continuous Galerkin method stabilized by interior penalty on the jumps of the gradients over interelement faces and the discontinuous Galerkin method with parametrized interior penalty stabilization both of the jumps of the function itself

and of its gradients over interior faces. We have reviewed the $h$-convergence analysis 


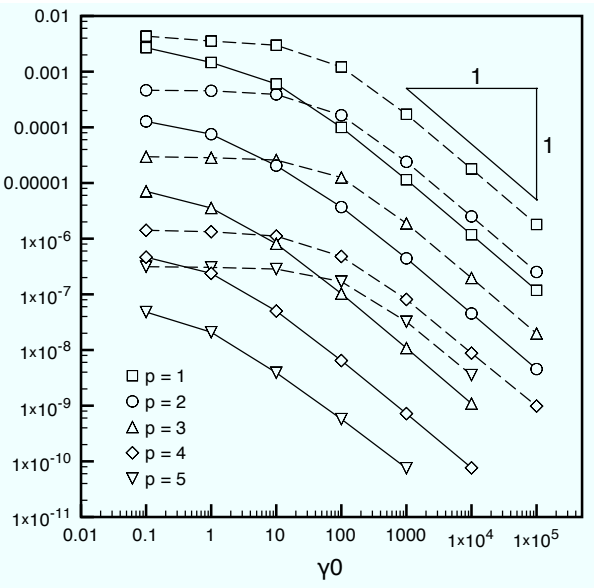

(a) $\left\|u_{c}-u_{d}\right\|_{L^{2}(\Omega)}$

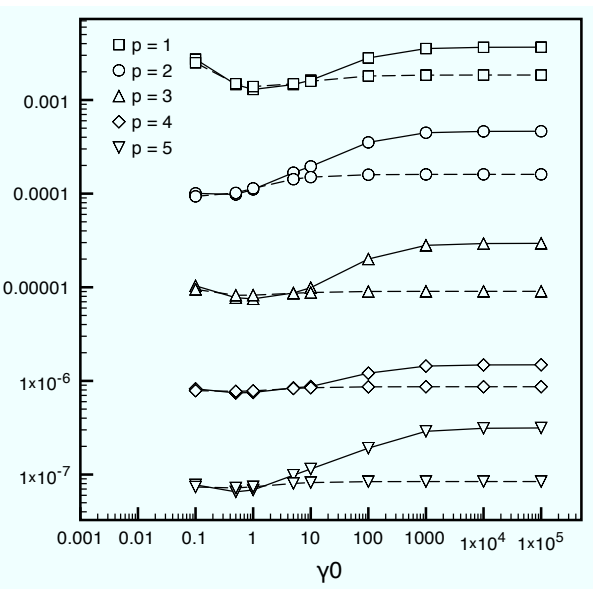

(b) $\left\|u-u_{d}\right\|_{L^{2}(\Omega)}$

FiguRE 4. Difference between the solutions of the discontinuous method and of the continuous method (a) and between the exact solution and the solution of the discontinuous method (b) for variable $\gamma_{0}$ and fixed $h$. The solid line corresponds to the choice $\gamma_{1}=0$ and the dashed line to the optimal choice of $\gamma_{1}>0$ according to figure 2.

for the continuous method with interior penalty and the augmented discontinuous method. We proved that the solution of the discontinuous method converges to the solution of the continuous method as the stabilization parameter of the interelement solution jump increases to infinity. This is also showed numerically together with some comparisons of the behavior of the interior penalty method using continuous and discontinuous approximations.

The techniques that we have advocated here for the stabilization of transport dominated advection-reaction problems can nowadays be regarded as efficient alternatives to the more classical upwind-based finite element approximations dated back to the pioneering work by Mitchell and Griffiths [24], the generalization and analysis by Baba and Tabata [1] or the fully consistent SUPG- or GLS-methods, see the pioneering work $[6,19]$ or the books $[27,30]$.

\section{ACKNOWLEDGEMENTS}

The authors acknowledge the financial support provided through the Swiss National Science Foundation under grant 200021 - 113304. This work has also been supported by Italian MIUR Cofin2005 "Numerical modelling in fluid dynamics with application to the cardiovascular system and environment".

\section{REFERENCES}

[1] K. Baba and M. Tabata. On a conservative upwind finite element scheme for convective diffusion equations. RAIRO Anal. Numér., 15(1):3-25, 1981. 
[2] I. Babuška and M. Zlámal. Nonconforming elements in the finite element method with penalty. SIAM J. Numer. Anal., 10:863-875, 1973.

[3] F. Brezzi, B. Cockburn, L. D. Marini, and E. Süli. Stabilization mechanisms in discontinuous Galerkin finite element methods. Comput. Methods Appl. Mech. Engrg., 195(25-28):32933310, 2006.

[4] F. Brezzi, P. Houston, D. Marini, and E. Süli. Modeling subgrid viscosity for advectiondiffusion problems. Comput. Methods Appl. Mech. Engrg., 190:1601-1610, 2000.

[5] F. Brezzi, L. D. Marini, and E. Süli. Discontinuous Galerkin methods for first-order hyperbolic problems. Math. Models Methods Appl. Sci., 14(12):1893-1903, 2004.

[6] A. N. Brooks and T. J. R. Hughes. Streamline upwind/Petrov-Galerkin formulations for convection dominated flows with particular emphasis on the incompressible Navier-Stokes equations. Comput. Methods Appl. Mech. Engrg., 32(1-3):199-259, 1982. FENOMECH '81, Part I (Stuttgart, 1981).

[7] E. Burman. A unified analysis for conforming and nonconforming stabilized finite element methods using interior penalty. SIAM J. Numer. Anal., 43(5):2012-2033 (electronic), 2005.

[8] E. Burman and A. Ern. Stabilized Galerkin approximation of convection-diffusion- reaction equations: discrete maximum principle and convergence. Math. Comp., 2005. accepted.

[9] E. Burman and A. Ern. Continuous interior penalty $h p$-finite element methods for advection and advection-diffusion equations. Math. Comp., 76:1119-1140, 2007.

[10] E. Burman and P. Hansbo. Edge stabilization for Galerkin approximations of convectiondiffusion-reaction problems. Comput. Methods Appl. Mech. Engrg., 193(15-16):1437-1453, 2004.

[11] E. Burman and B. Stamm. Minimal stabilization of discontinuous Galerkin finite element methods for hyperbolic problems. J. Sci. Comput., 2007.

[12] E. Burman and P. Zunino. A domain decomposition method based on weighted interior penalties for advection-diffusion-reaction problems. SIAM Journal on Numerical Analysis, 44(4):1612-1638, 2006.

[13] J. Douglas and T. Dupont. Interior penalty procedures for elliptic and parabolic Galerkin methods. In Computing methods in applied sciences (Second Internat. Sympos., Versailles, 1975), pages 207-216. Lecture Notes in Phys., Vol. 58. Springer, Berlin, 1976.

[14] A. Ern and J.-L. Guermond. Discontinuous Galerkin methods for Friedrichs' systems. I. General theory. SIAM J. Numer. Anal., 44(2):753-778 (electronic), 2006.

[15] J. L. Guermond. Stabilization of Galerkin approximations of transport equations by subgrid modeling. M2AN Math. Model. Numer. Anal., 33(6):1293-1316, 1999.

[16] R. H. W. Hoppe and B. Wohlmuth. Element-oriented and edge-oriented local error estimators for non-conforming finite element methods. Math. Model. Numer. Anal. (M2AN), 30:237-263, 1996.

[17] P. Houston, C. Schwab, and E. Süli. Discontinuous $h p$-finite element methods for advectiondiffusion-reaction problems. SIAM J. Numer. Anal., 39(6):2133-2163 (electronic), 2002.

[18] T. J. R. Hughes, G. Engel, L. Mazzei, and M. G. Larson. The continuous Galerkin method is locally conservative. J. Comput. Phys., 163(2):467-488, 2000.

[19] T. J. R. Hughes, L. P. Franca, and G. M. Hulbert. A new finite element formulation for computational fluid dynamics. VIII. The Galerkin/least-squares method for advective-diffusive equations. Comput. Methods Appl. Mech. Engrg., 73(2):173-189, 1989.

[20] C. Johnson and J. Pitkäranta. An analysis of the discontinuous Galerkin method for a scalar hyperbolic equation. Math. Comp., 46(173):1-26, 1986.

[21] O. Karakashian and F. Pascal. A-posteriori error estimates for a discontinuous Galerkin approximation of second order elliptic problems. SIAM J. Numer. Anal., 41(6):2374-2399, 2003.

[22] M. Larson and A. Niklasson. Conservation properties for the continuous and discontinuous Galerkin method. Technical Report 2000-08, Chalmers Finite Element Center, Chalmers University, 2000.

[23] P. Lesaint and P. A. Raviart. On a finite element method for solving the neutron transport equation. In Mathematical aspects of finite elements in partial differential equations (Proc. Sympos., Math. Res. Center, Univ. Wisconsin, Madison, Wis., 1974), pages 89-123. Publication No. 33. Math. Res. Center, Univ. of Wisconsin-Madison, Academic Press, New York, 1974 . 
[24] A. R. Mitchell and D. F. Griffiths. Upwinding by Petrov-Galerkin methods in convectiondiffusion problems. J. Comput. Appl. Math., 6(3):219-228, 1980.

[25] C. Prud'homme. A domain specific embedded language in $\mathrm{C}++$ for automatic differentiation, projection, integration and variational formulations. Scientific Programming, 14(2):81-110, 2006.

[26] C. Prud'homme. Life: Overview of a unified C++ implementation of the finite and spectral element methods in 1d, 2d and 3d. In Workshop On State-Of-The-Art In Scientific And Parallel Computing, Lecture Notes in Computer Science, page 10. Springer-Verlag, 2006. Accepted.

[27] A. Quarteroni and A. Valli. Domain decomposition methods for partial differential equations. Numerical Mathematics and Scientific Computation. The Clarendon Press Oxford University Press, New York, 1999. , Oxford Science Publications.

[28] W. H. Reed and T. R. Hill. Triangular mesh methods for the neutron transport equation. Technical Report LA-UR-73-479, Los Alamos Scientific Laboratory, 1973.

[29] A. Romkes, S. Prudhomme, and J. T. Oden. A priori error analyses of a stabilized discontinuous Galerkin method. Comput. Math. Appl., 46(8-9):1289-1311, 2003.

[30] H. G. Roos, M. Stynes, and L. Tobiska. Numerical methods for singularly perturbed differential equations, volume 24 of Springer Series in Computational Mathematics. Springer-Verlag, Berlin, 1996. Convection-diffusion and flow problems.

Erik Burman, EPFL, IACS-CMCS, Station 8, 1015 Lausanne, Switzerland;

Department of Mathematics, Mantell Bullding, University of Sussex, Falmer, Brighton, BN1 9RF, United Kingdom

E-mail address: E.N.Burman@sussex.ac.uk

Alfio Quarteroni, EPFl, IACS-CMCS, Station 8, 1015 Lausanne, Switzerland; MOX-Dipartimento di Matematica "F. Brioschi", Politecnico di Milano, 20133 Milano, ITALY

E-mail address: alfio.quarteroni@epfl.ch

Benjamin Stamm, EPFL, IACS-CMCS, Station 8, 1015 Lausanne, Switzerland

E-mail address: benjamin.stamm@epfl.ch 